\title{
Discrimination of Real and Virtual High-Definition Textured Surfaces
}

\author{
Hong Z. Tan ${ }^{1}$, Bernard D. Adelstein ${ }^{2}$, Ryan Traylor $^{1}$, Matthew Kocsis ${ }^{1}$ and E. Dan Hirleman ${ }^{3}$ \\ ${ }^{1}$ Haptic Interface Research Laboratory \\ ${ }^{3}$ School of Mechanical Engineering \\ Purdue University, West Lafayette, IN 47907 \\ \{hongtan, traylorr, mkocsis, hirleman\}@purdue.edu \\ ${ }^{2}$ Human Factors Division, NASA Ames Research Center, Moffett Field, CA 94035 \\ \{bda@eos.arc.nasa.gov\}
}

\begin{abstract}
Research on haptic texture perception requires the availability of textured surfaces with high precision and fine resolution. Given the exquisite sensitivity of the human fingers to minute differences in surface details, the textured surfaces need to be precisely defined to a micron level. Real and virtual high-definition textured surfaces were used in the present study of amplitude discrimination of sinusoidal gratings. Similar thresholds were obtained despite the difference in contact modes (fingertip on real textures and a point on virtual textures). The results support the use of high position-resolution force-feedback devices in studying texture perception, especially in situations where the fabrication of real textures is either beyond the resolution of the fabrication process or simply too expensive and time-consuming.
\end{abstract}

KEYWORDS: texture perception, real texture, virtual texture, discrimination.

\section{INTRODUCTION}

Haptic texture perception is a fascinating phenomenon that has attracted much research attention since perhaps 1925 (Katz, 1925/1989). In many ways, haptic texture perception is akin to visual color perception in the sense that there seems to be an infinite variety of textures to the touch, as there are colors to the eyes, in the natural world. What would it take then for us to manipulate the feel of surface textures as easily as we can the color of images? Key issues to be resolved are the physical determinants, the perceptual dimensionality, and the neural mechanisms of texture perception (Connor, Hsiao, Phillips, \& Johnson, 1990). A multidimensional scaling study using seventeen texture patches (sandpaper, velvet, etc.) found that a threedimensional perceptual space provided a satisfactory representation of perceived similarity judgment data (Hollins, Faldowski, Rao, \& Young, 1993). The dimensions were roughness-smoothness, hardness-softness, and possibly "springiness" of the texture surfaces.

To study the physical determinants and the neural mechanisms of texture perception, texture samples with well-controlled surface features are needed. Of the three dimensions proposed by Hollins et al. (1993), much is now known about the roughness-smoothness dimension (see Klatzky, Lederman, Hamilton, Grindley, \&

Symposium on Haptic Interfaces for

Virtual Environment and Teleoperator Systems 2006

March 25 - 26, Alexandria, Virginia, USA

1-4244-0226-3/06/\$20.00 O2006 IEEE
Swendsen, 2003, for a recent review). Typical stimuli used for roughness studies include sandpapers with varying grit numbers (e.g., Stevens \& Harris, 1962), metal plates with linear grooves (e.g., Lederman, 1974; Lederman \& Taylor, 1972), and raised dots with controlled height and density (e.g., Connor \& Johnson, 1992; Lederman, Klatzky, Hamilton, \& Ramsay, 1999; Lederman, Thorne, \& Jones, 1986).

An alternative is to use one-dimensional sinusoidal gratings with controlled amplitude and spatial wavelength, since sinusoidal signals can be viewed as the building blocks of any surface profile. It is however extremely expensive and time-consuming to fabricate high-definition (i.e., high-precision, fine-resolution) sinusoidal surfaces. We are aware of only one prior study that used such stimuli (see Nefs, Kappers, \& Koenderink, 2001). On the other hand, it is straightforward to render micro-geometric features such as virtual sinusoidal (or square, etc.) gratings using softwareprogrammable force-feedback devices (e.g., Choi \& Tan, 2005; Ho, Adelstein, \& Kazerooni, 2004; Weisenberger, Krier, \& Rinker, 2000). Haptic interfaces are similarly well-suited for modulating other perceptual features such as Hollins et al.'s (1993) hardness/softness and springiness (e.g., Rosenberg \& Adelstein, 1993; Lawrence, Pao, Dougherty, Salada, \& Pavlou, 2000).

It is much more desirable to use virtual textures due to the relative ease with which the parameters of the textured surfaces can be altered so that more efficient experimental methods such as adaptive procedures can be used. To do so requires that the following two issues be resolved first:

- What does it mean for real or virtual textures to be "highdefinition" enough for studying human perception of texture?

- How valid is it to use virtual textures instead of real ones for studying human texture perception?

If the goal is to measure the amplitude discrimination threshold for sinusoidal gratings, then the answer to the first question is that the resolution of the textured surfaces needs to be on the order of single microns (Nefs et al., 2001). Lederman's study clearly demonstrated the influence of microscale surface structure on perception (Lederman, 1974). Two aluminum surfaces with identical square-grating parameters (groove width $=0.125 \mathrm{~mm}$; land width $=0.25 \mathrm{~mm}$ ) were fabricated using a cutting bit and an electric discharge machining (EDM) technique, respectively. The surfaces looked quite different under a scanning electron microscope (see Figure 1) and produced different perceived 

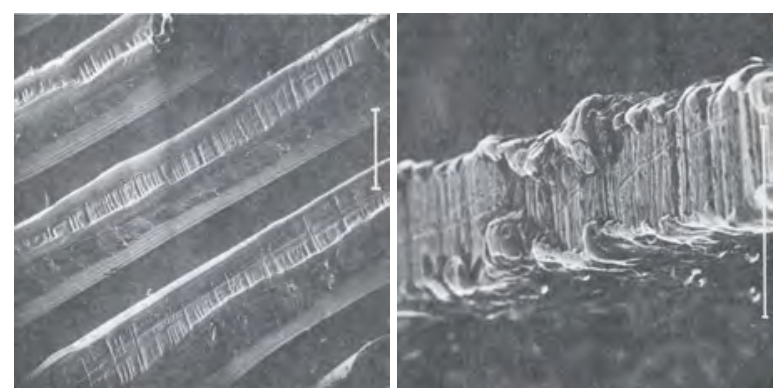

Figure 1. Scanning electron micrographs of two aluminum plates with grooves cut with a cutting bit (left) and with a process called electric discharge machining (right). The vertical white lines represent $200 \mu \mathrm{m}$ each. From Lederman (1974) (Figs. 5 and 6).

roughness curves as a function of nominal groove width. In the present study, stainless steel texture samples were fabricated using the EDM technique with an additional polishing step to ensure the smoothness of the resulting sinusoidal gratings. Surface profilometry was used to verify these real textured surfaces. For virtual surface rendering, it should be noted that most commercially-available force feedback devices have a position resolution in the range 10-60 $\mu \mathrm{m}$ (e.g., the PHANToM by SensAble Technologies has a nominal position resolution of 30 $\mu \mathrm{m}$; the Omega by ForceDimension has a resolution of $9 \mu \mathrm{m})$. In the present study, we used a custom-designed force-feedback device with a resolution at or below $1.0 \mu \mathrm{m}$. Note that with all these devices, because of mechanism compliance and other imperfections, the control resolution will be to some extent poorer.

To address the second question of whether virtual textures can be used to replace real textures in studying texture perception, we compared discrimination thresholds obtained with real and virtual textures. Ideally, one would prefer to render virtual textures that cannot be distinguished from real textures. It is however almost impossible to demonstrate the equivalence experimentally, short of making interfaces to real and virtual objects that to the research participants feel, look, and sound identical. Nevertheless, several studies have compared user performance using similar tasks implemented in real and virtual environments, and the results are mixed. For example, Buttolo, Kung and Hannaford (1995) reported almost identical task completion time in direct and virtual manipulations. In another study by Unger et al. (2002), an interface for a real peg-in-hole task was built to be like the interface to a magnetic levitation haptic device, and it was found that participants performed faster inserting a real peg in a real hole than using the maglev device in a virtual environment. Metrics other than task completion time have also been used for comparing performance in real and virtual environments. West and Cutkosky (1997) asked users to count the number of 1-D sinusoidal cycles on real and virtual surfaces. The best accuracy was found using fingertips on real textured surfaces, followed by using a stylus on real textures, and with the lowest accuracy associated with using a stylus on virtual textures. Greenish et al. (2002) demonstrated similar tissue identification accuracy with a real tissue-cutting task and with a simulated cutting task where force data recorded during real cutting were played back through a custom-designed haptic device. Most recently, O’Malley and Goldfarb (2005) concluded that size-identification tasks performed with a haptic interface capable of sufficient force output can approach the percent-correct level in a real environment, but the accuracy of size-discrimination tasks performed in a virtual environment was consistently lower than that in a real environment.

In the present study, we examined whether similar texture discrimination thresholds can be obtained from high-definition real and virtual surfaces using nearly identical experimental methods. We chose the discrimination threshold as a performance metric because it is a standard measure in the psychophysical literature and therefore its value can be readily compared to those available in the literature. Two experimental conditions, exploring real textured surfaces with the fingertip and stroking virtual surfaces with a stylus, were employed. We did not use a third condition of stroking virtual surfaces with a stylus in this initial study because we expected the threshold value associated with this condition to be between those from the first two conditions (cf. West \& Cutkosky, 1997). To the extent that we obtain similar thresholds with the two conditions employed in the present study, we will be able to conclude that texture discrimination performance is similar using real and virtual textured surfaces, without having tested the third condition.

\section{Methods}

This section describes the methods that are common to both experiments. Details that are specific to each experiment are included later in Sections 3 and 4.

\subsection{Stimuli}

The textured surfaced used in the experiments were onedimensional sinusoidal gratings with a fixed spatial wavelength of $2.5 \mathrm{~mm}$. The amplitudes of the gratings ( $1 / 2$ peak-to-trough) were $50 \mu \mathrm{m}\left(\mathrm{A}_{0}\right.$, reference), $55 \mu \mathrm{m}\left(\mathrm{A}_{1}\right), 60 \mu \mathrm{m}\left(\mathrm{A}_{2}\right), 65 \mu \mathrm{m}\left(\mathrm{A}_{3}\right)$, and $70 \mu \mathrm{m}\left(\mathrm{A}_{4}\right)$.

\subsection{Procedures}

The experimental procedure used a two-interval two-alternative forced-choice paradigm. There were four pairs of stimuli: $A_{0}$ and $\mathrm{A}_{1}, \mathrm{~A}_{0}$ and $\mathrm{A}_{2}, \mathrm{~A}_{0}$ and $\mathrm{A}_{3}$, and $\mathrm{A}_{0}$ and $\mathrm{A}_{4}$, with $\mathrm{A}_{0}$ serving as the reference in all pairs. The same texture pair was used within each run with 50 trials/run for Exp. I and 100 trials/run for Exp. II. The participant could examine both of the textures presented during each trial. The stimulus was called " 1 " if the texture with the higher amplitude was on the left, and " 2 " if the reference texture $\left(\mathrm{A}_{0}\right)$ was on the left. A total of 200 trials were conducted for each pair of stimuli. The order of the 16 runs in Exp. I ( 4 pairs $\times 4$ runs/pair) or the 8 runs in Exp. II ( 4 pairs $\times 2$ runs/pair) was randomized for each participant. The experiment was spread over several sessions with each session lasting between one and two hours. A computer program determined the stimulus to be presented on each trial ("1" or " 2 "), and collected the participant's responses ("1" or "2"). 


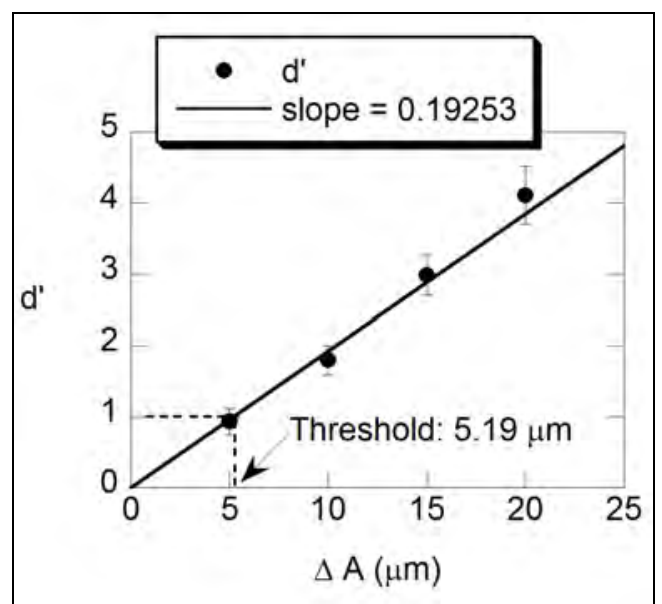

Figure 2. Calculation of discrimination threshold from $\mathrm{d}^{\prime}$ scores. Shown are the $d^{\prime}$ values $( \pm$ std. dev.) associated with the four stimulus pairs, plotted against $\Delta \mathbf{A}$, the amplitude difference between a pair of textured surfaces. Also shown are the fitting line with the average slope and the threshold.

The participants were instructed to stroke the textured surfaces laterally along the sinusoidal gratings. They used the tip of the index fingerpad of the dominant hand for real textures (Exp. I) and (the virtual tip of) a stylus for virtual textures (Exp. II). There was no limit on length of stroke or how many times the participant stroked the two textured surfaces. Trial-by-trial correct-answer feedback was provided. During both experiments, headphonestyle hearing protectors (Twin Cup H10A, NRR 29; Peltor, Sweden) were worn by all participants.

Training was provided at the beginning of each run. Data collection began when the participant was ready.

\subsection{Data Analysis}

For each participant, a stimulus-response matrix for each stimulus pair was constructed and the sensitivity index $d^{\prime}$ and the response bias $\beta$ were calculated (Macmillan \& Creelman, 2004). To estimate the texture discrimination threshold (sometimes called JND - just noticeable difference) from $d^{\prime}$ values, we first calculated the slope of the best-fitting line by averaging the slopes from the $d^{\prime}$ values corresponding to the four stimulus pairs:

$$
k=\left(d_{1}^{\prime} / 5+d_{2}^{\prime} / 10+d_{3}^{\prime} / 15+d^{\prime}{ }_{4} / 20\right) / 4 .
$$

The threshold was then taken as the amplitude difference that corresponded to $d^{\prime}=1$; i.e., $1 / k$ (see Pang, Tan, \& Durlach, 1991, for details). Figure 2 shows an example of data processing using data for one subject from Exp. II.

\section{EXP. I: DISCRIMINATION OF REAL TEXTURES}

\subsection{Participants}

Four participants, two males and two females, took part in Exp. I. Two of the participants are co-authors of this article, and had participated in several pilot runs before data collection began. All participants were right-handed and reported no conditions that would compromise their sense of touch.

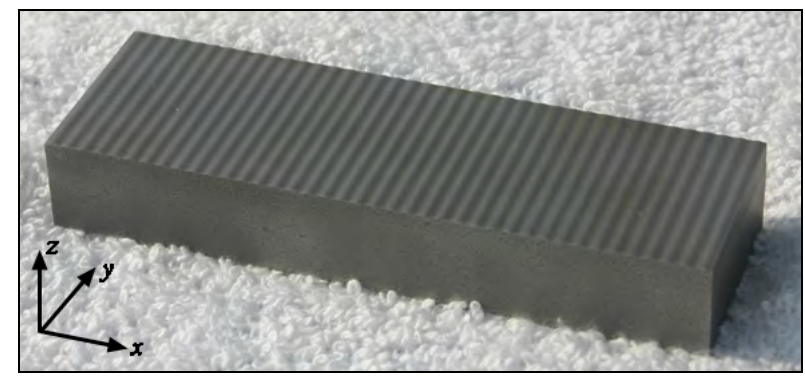

Figure 3. Texture sample with $A_{1}=55 \mu \mathrm{m}$.

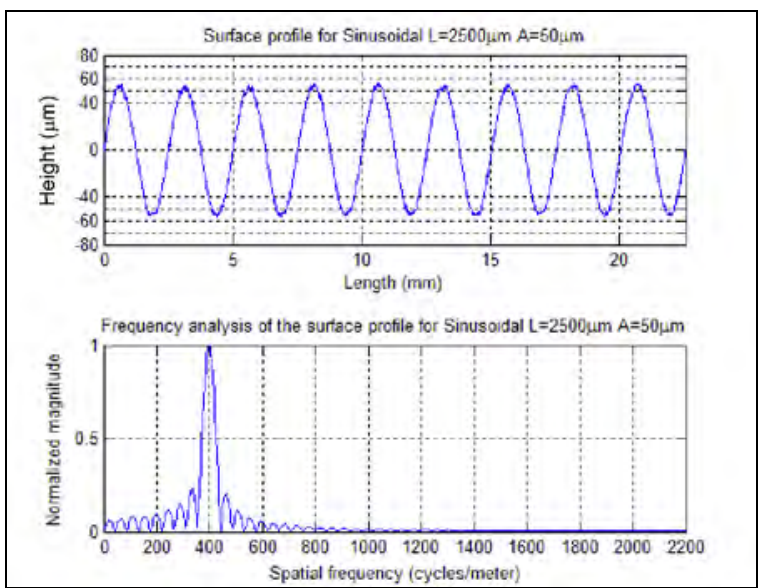

Figure 4. (top) Surface height along the length of the reference texture sample with $A_{0}=\mathbf{5 0} \mu \mathrm{m}$. (bottom) Fourier transform of the height trace shown on the top. There were no discernable harmonics at multiples of 400 cycles/meter. The side lobes were associated with the sinc function due to the finite length of the top trace.

\subsection{Validation of Texture Samples}

The texture samples were machined out of stainless steel blocks using a wire electric discharge machining (EDM) process (Figure 3). The dimension of each block was $100 \mathrm{~mm}(\mathrm{~L}) \times 30 \mathrm{~mm}(\mathrm{~W}) \times$ $15 \mathrm{~mm}(\mathrm{H})$.

A surface profilometer (model Surtronic 3plus, Taylor Hobson, England) was used to validate the surface geometry of each of the five sinusoidal texture samples. The tip of the profilometer was moved along the length of the block while maintaining contact with the textured surface. The height trace $h(x)$ along a fixed $y$ position could then be plotted and analyzed. Figure 4 shows the $h(x)$ plot for the reference texture sample and its Fourier transform. It can be seen that the EDM produced a very "clean" sinusoidal profile with amplitude of $50 \mu \mathrm{m}$. There were no harmonics indicated by the Fourier spectrum.

The profilometer was also used to measure parallel profiles along several $y$ values to check the texture consistency along the width of the block. The traces were then combined to form three dimensional (3D) raster-scan images of each texture sample. Figure 5 shows one plot for $\mathrm{A}_{3}=65 \mu \mathrm{m}$. Further Fourier analysis confirmed that the surface profile was consistent along its width. 


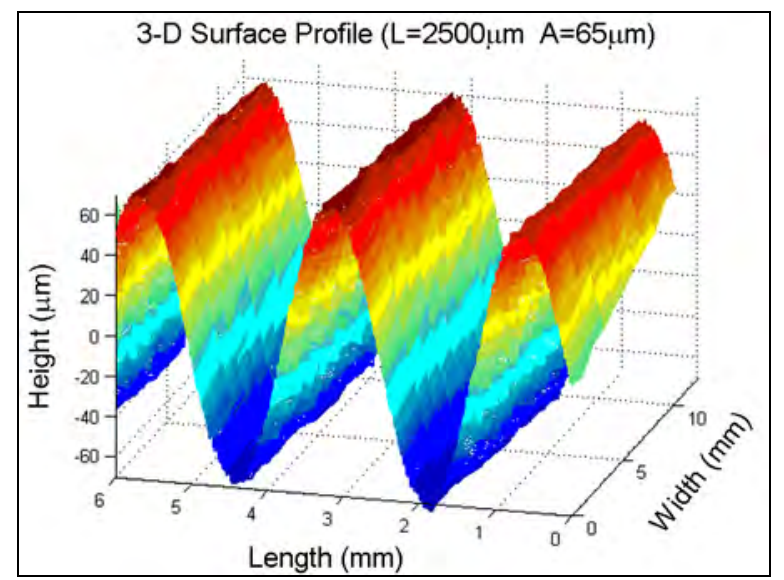

Figure 5. 3-D raster image of the surface of the texture sample with a sinusoidal amplitude of $65 \mu \mathrm{m}$. Multiple length-wise profilometer scans taken at several positions along the width are combined to form the image.

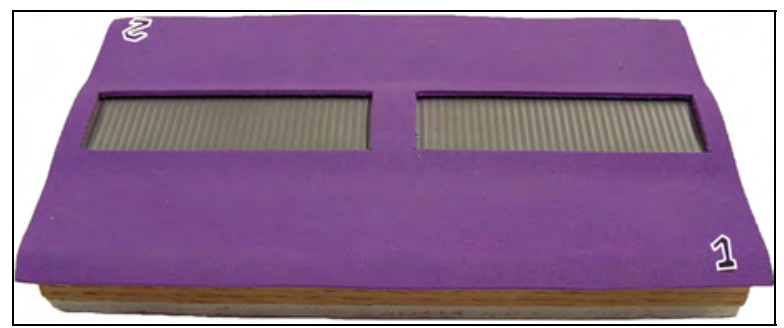

Figure 6. Test apparatus used in Exp. I. It housed two stainless steel texture samples. The numbers " 1 " and "2" were used by the experimenter to determine the orientation at which the texture samples were presented to the participant.

\subsection{Procedure}

Before the experiment, participants were instructed to wash their hands with soap to remove residual oils on the skin surfaces. The experimenter and the participant were separated by a curtain and were seated facing each other across a table. The participant had to slide the dominant hand under the curtain in order to stroke with the index fingertip texture samples that were hidden from the participant's view. Before each run, the experimenter inserted the two textured blocks corresponding to the stimulus pair into a long slot. A piece of felt fabric with two openings slightly smaller than the textured surfaces (so as to mask the edges) covered the test apparatus (see Fig. 6). For each trial, a computer program determined which stimulus should be presented. The experimenter spun the entire setup on the table top several times regardless of whether the stimulus had changed from the previous trial. The experimenter then stopped the setup at the desired orientation so that the texture with the higher amplitude was either on the left side (stimulus " 1 ") or on the right side (stimulus "2") as viewed by the participant.

\subsection{Results}

Table 1 shows the texture discrimination thresholds from Exp. I. On average, the participants were extremely sensitive to the
Table 1. Texture discrimination thresholds from Exp. I

\begin{tabular}{|c|c|c|}
\hline Participant & Threshold $(\mu \mathrm{m})$ & \multirow{2}{*}{ Avg \pm SD $(\mu \mathrm{m})$} \\
\hline 1 & 4.15 & \multirow{3}{*}{$3.90 \pm 0.77$} \\
\hline 2 & 4.41 & \\
\hline 3 & 4.27 & \\
\hline 4 & 2.75 & \\
\hline
\end{tabular}

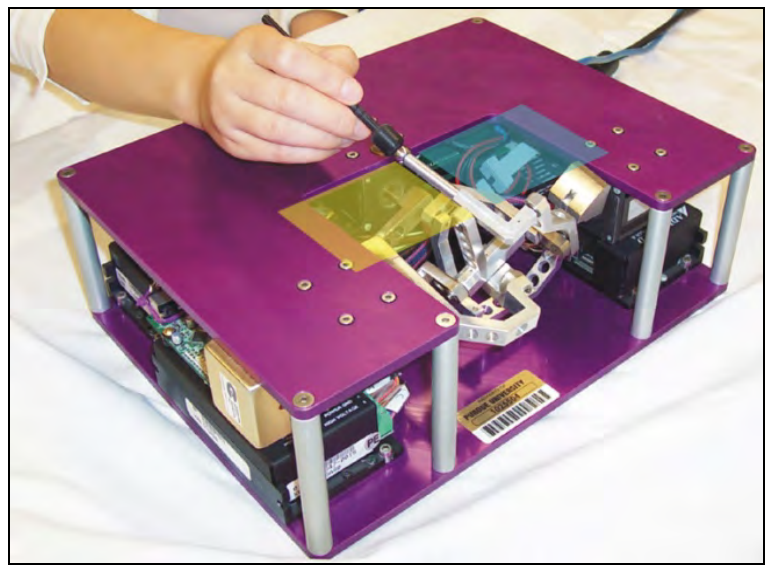

Figure 7. The 3-DOF force-feedback device used to render virtual textures in Exp. II. The cyan and yellow patches indicate (roughly) the locations of the two virtual textured surfaces.

amplitude difference between two sinusoidal surface profiles. The average discrimination threshold was as small as $3.90 \mu \mathrm{m}$ for a reference amplitude of $50 \mu \mathrm{m}$. After the experiments, the participants commented that a great deal of concentration was needed to perform the task of discriminating between texture samples that felt almost identical to the fingertip.

\section{EXP. II: DISCRIMINATION OF VIRTUAL TEXTURES}

\subsection{Participants}

Ten participants, five males and five females, took part in Exp. II. They were right-handed and reported no conditions that would compromise their sense of touch. Only one participant took part in both experiments.

\subsection{Virtual Textures}

The virtual textures were rendered by a 3-DOF force-feedback joystick called the ministick (Traylor, Wilhelm, Adelstein, \& Tan, 2005), based on the metal linkage described by Steger, Lin, Adelstein, and Kazerooni (2004), but with a revised, PC-resident control and simulation architecture (Traylor et al., 2005). The ministick has a typical position resolution of $1 \mu \mathrm{m}$. Its force commands are updated at $2 \mathrm{kHz}$.

The layout of the virtual textures was very similar to that of the stainless steel texture blocks used in Exp. I (compare Fig. 6 and 7). The height map was defined by

$$
h(x)=A \sin (2 \pi x / L)+A,
$$


Table 2. Texture discrimination thresholds from Exp. II

\begin{tabular}{|c|c|c|}
\hline Participant & Threshold $(\mu \mathrm{m})$ & Avg \pm SD $(\mu \mathrm{m})$ \\
\hline 1 & 4.86 & \\
\hline 2 & 5.66 & \\
\hline 3 & 5.12 & \\
\hline 4 & 4.48 & \multirow{5}{*}{5.05} \\
\hline 5 & 5.19 & \multirow{2}{*}{ \pm 1.07} \\
\hline 6 & 3.64 & \\
\hline 7 & 7.56 & \\
\hline 8 & 4.28 & \\
\hline 9 & 4.32 & \\
\hline 10 & 5.34 & \\
\hline
\end{tabular}

where $L=2.5 \mathrm{~mm}$, and $A$ was selected on the fly from the five amplitude values of $50,55,60,65$, or $70 \mu \mathrm{m}$. The restoring force was calculated as

$$
F=\left\{\begin{array}{cc}
K \times\left[h(x)-p_{z}\right] & \text { when } p_{z}<h(x) \\
0 & \text { o.w. }
\end{array}\right.
$$

where the restoring force $F$ always pointed up, $p_{z}$ was the $z$ position of the stylus tip, and the stiffness constant $K$ was 2.0 $\mathrm{N} / \mathrm{mm}$. Note that this is the simple "slippery" model provided with the PHANToM (Massie, 1996), not a more sophisticated geometric model such as that proposed by Ho, Basdogan, and Srinivasan (1999). As shown in Fig. 7, two virtual texture patches with a gap of $12.7 \mathrm{~mm}(0.5 \mathrm{in})$ were presented on each trial. The participant's task was to indicate which virtual texture patch had the higher amplitude.

\subsection{Procedure}

The experimental procedure in Exp. II was essentially the same as that in Exp. I with one exception. Because it was faster to present the virtual textures than the real ones, each run in Exp. II consisted of 100 trials instead of the 50-trial runs used in Exp. I.

\subsection{Results}

Texture discrimination thresholds from Exp. II are shown in Table 2. The average discrimination threshold was $5.05 \mu \mathrm{m}$ for a $50 \mu \mathrm{m}$ reference amplitude.

\section{Discussion}

Two experiments to estimate the amplitude discrimination thresholds for sinusoidal gratings were conducted using highdefinition real and virtual haptic textures. Exp. I employed stainless steel textured surfaces produced with an EDM process that included post-fabrication polishing. The average $(N=4)$ discrimination threshold was $3.90 \mu \mathrm{m}$, or $7.8 \%$ of the reference amplitude of $50 \mu \mathrm{m}$. Our result can be compared directly with that obtained by Nefs et al. (2001) where similar stimuli and methods were used. Specifically, they found a threshold of $8 \mu \mathrm{m}$ for sinusoidal gratings with an amplitude of $51.2 \mu \mathrm{m}$ and a spatialperiod of $2.5 \mathrm{~mm}$. The relatively lower threshold found in the present study may be the result of a small difference in the instructions given to the participants. In the Nefs et al. (2001) study, the participants could only stroke the textured surfaces twice from side to side, whereas in the present study participants were allowed to stroke the surfaces as many times as they wished. In our pilot tests, we had tried to limit the number of strokes to two, but found the task to be too difficult to perform. We then decided to let the participants feel the textures for as long as needed to measure the best achievable thresholds (personal communications with R. Klatzky and S. Lederman, 2004). Therefore, the $3.90 \mu \mathrm{m}$ result from our present Exp. I can be viewed as a lower-bound threshold for amplitude discrimination of sinusoidal gratings with $50 \mu \mathrm{m}$ amplitude and $2.5 \mathrm{~mm}$ spatial-period. The $8 \mu \mathrm{m}$ value from Nefs et al. (2001) can be considered a typical threshold for amplitude discrimination of similar sinusoidal (51.2 $\mu \mathrm{m}$ amplitude) gratings.

In Exp. II, a high displacement resolution force-feedback ministick was used to simulate virtual textures having the same surface profiles as those in Exp. I. An average $(N=10)$ amplitude discrimination threshold of $5.05 \mu \mathrm{m}(10.1 \%$ of the reference amplitude of $50 \mu \mathrm{m}$ ) was found. This value can be compared to results from a series of experiments on haptic texture perception using force-feedback devices (Poling \& Weisenberger, 2004; Weisenberger \& Krier, 1997; Weisenberger, Krier, \& Rinker, 1998; Weisenberger et al., 2000; Weisenberger, Krier, Rinker, \& Kreidler, 1999; West \& Cutkosky, 1997). Our Exp. II is similar to Weisenberger and Krier's (1997) study where 2-D sinusoidal "bumps" were simulated on a PHANToM force-feedback device. They estimated amplitude JNDs over a range of amplitude and spatial frequency values. For gratings with a spatial frequency of 3.88 cycles/cm, which is close to the $2.5 \mathrm{~mm}$ wavelength (4 cycles $/ \mathrm{cm}$ ) of our 1-D gratings in the present study, they found JNDs of roughly $0.15 \mathrm{~mm}(25-12.5 \%$ of the reference) over a reference amplitude range of $0.6-1.2 \mathrm{~mm}$. Thus, when expressed as Weber fractions, the results from Weisenberger and Krier (1997) and our Exp. II are roughly comparable considering the many differences in stimuli, apparatus and experimental procedures between the two studies.

A key difference between the two experiments in our present study was the surface contact mode. The real texture was explored with the bare finger, which conveys spatial-intensive information as well as vibration frequency arising from the speed of motion across the grating. During normal active touch (i.e., during volitional limb movement), observers rely mainly on spatialintensive information and can ignore vibratory frequencies to judge texture intensity (i.e., roughness) (Lederman et al., 1999). The virtual texture, on the other hand, can only be experienced via the mechanical vibration of the haptic interface linkage produced by the interaction between the stylus's virtual tip and texture as the grating is stroked. As is the case for exploring real textures with a rigid stylus, observers can make use of vibratory sensations caused by stylus-grating interaction in conjunction with the kinesthetic feedback from their movement to judge texture intensity (Lederman et al., 1999). Furthermore, the touching of real and virtual surfaces is inherently different. Specifically, a real stainless steel surface has an almost infinite stiffness and cannot be penetrated by the fingertip or a probe, whereas an impedance 
implementation of a virtual surface has a limited stiffness and has to be penetrated by a virtual probe before it can be perceived. In light of these differences, we conclude that the thresholds for Exp. I $(3.90 \mu \mathrm{m})$ and Exp. II $(5.05 \mu \mathrm{m})$ are remarkably similar.

Klatzky and Lederman (1999) demonstrated that participants perceived raised dot textures with smaller inter-element (i.e., dot) spacing as being rougher when experienced with a smaller than a larger diameter probe tip, and least rough with the bare finger tip. But, as inter-element spacing was increased, the ordering of perceived roughness by probe sizes and fingertip was reversed. Equating inter-element spacing between raised dots with the spacing between our textures' ridges would indicate that the crossover in Klatzky and Lederman's roughness ordering occurred approximately at the wavelength of our sinusoidal gratings.

While increased subjective roughness ratings in Klatzky and Lederman's study can be expected to correspond to higher sensitivity and lower threshold, predicting whether the finger or stylus should produce more acute (i.e., smaller) thresholds in our present experiments is difficult for two reasons. First, as noted above, there is a probe tip diameter dependent crossover in roughness ratings (Klatzky \& Lederman, 1999; Lederman et al., 1999), suggesting that threshold magnitudes should be comparable for the finger and probe (stylus) near the wavelength of our textures. Second, the probe tip in our second experiment's virtual interface is modeled as a simple point and therefore has zero diameter. Extrapolating from the two probe tip diameters in Klatzky and Lederman's (1999) study, one might expect the roughness rating crossover point to shift to a lower inter-element spacing than our present grating wavelength. Such a shift would make the perceived roughness greater and, therefore, the threshold lower for the finger than for the virtual probe.

Given the similarity between the amplitude thresholds obtained in the present Exps. I and II, the ministick haptic interface device appears to be an appropriate device for studying haptic texture perception. The ability to simulate haptic textures opens the door to many new experiments that can be conducted more efficiently using adaptive procedures (see also discussion on experiments for testing haptic "critical bandwidth" in Nefs, Kappers, \& Koenderink, 2003). To more precisely assess the validity of our approach, future work should compare stylus-mediated perception of real and virtual textures. With the appropriate device and interaction algorithm, a virtual haptic interface can enable rapid prototyping of the geometry and dynamics of not only textures but also of the probe tip.

\section{ACKNOWLEDGement}

This work was supported in part by a National Science Foundation Award under Grant No. 0098443-IIS, and in part by NASA under award no. NCC 2-1363. BDA was supported by NASA's Space Human Factors Engineering Program (131-20-30). The authors wish to thank Drs. Susan Lederman and Roberta Klatzky for discussions on experimental methods, and Mike Sherwood at Purdue University for his assistance with the fabrication of the stainless steel texture samples used in Exp. I.

\section{REFERENCES}

Buttolo, P., Kung, D., \& Hannaford, B. (1995). Manipulation in Real, Virtual, and Remote Environments. Proceedings of the IEEE International Conference on Systems, Man, and Cybernetics, 5, 46564661.

Choi, S., \& Tan, H. Z. (2005). Perceived instability of virtual haptic texture. II. Effect of collision detection algorithm. Presence: Teleoperators and Virtual Environments, 14(4), 463-481.

Connor, C. E., Hsiao, S. S., Phillips, J. R., \& Johnson, K. O. (1990). Tactile roughness: Neural codes that account for psychophysical magnitude estimates. Journal of Neuroscience, 10, 3823-3836.

Connor, C. E., \& Johnson, K. O. (1992). Neural coding of tactile texture: Comparison of spatial and temporal mechanisms for roughness perception. Journal of Neuroscience, 12, 3414-3426.

Greenish, S., Hayward, V., Chial, V., Okamura, A., \& Steffen, T. (2002). Measurement, analysis, and display of haptic signals during surgical cutting. Presence: Teleoperators and Virtual Environments, 11(6), 626-651.

Ho, C., Basdogan, C., \& Srinivasan, M. A. (1999). Efficient point-based rendering techniques for haptic display of virtual objects. Presence, $8(5), 477-491$.

Ho, P. P., Adelstein, B. D., \& Kazerooni, H. (2004). Judging 2D versus 3D square-wave virtual gratings. Proceedings of the 12th International Symposium on Haptic Interfaces for Virtual Environment and Teleoperator Systems (HAPTICS '04), 176- 183.

Hollins, M., Faldowski, R., Rao, S., \& Young, F. (1993). Perceptual dimensions of tactile surface texture: A multidimensional scaling analysis. Perception \& Psychophysics, 54(6), 697-705.

Katz, D. (1925/1989). The World of Touch. Hillsdale, NJ: Lawrence Erlbaum Associates.

Klatzky, R. L., \& Lederman, S. J. (1999). Tactile roughness perception with a rigid link interposed between skin and surface. Perception \& Psychophysics, 61(4), 591-607.

Klatzky, R. L., Lederman, S. J., Hamilton, C., Grindley, M., \& Swendsen, R. H. (2003). Feeling textures through a probe: Effects of probe and surface geometry and exploratory factors. Perception \& Psychophysics, 65(4), 613 - 631.

Lawrence, D. A., Pao, L. Y., Dougherty, A. M., Salada, M. A., \& Pavlou, Y. (2000). Rate-hardness: A new performance metric for haptic interface. IEEE Transactions on Robotics and Automation, 16(4), 357371.

Lederman, S. J. (1974). Tactile roughness of grooved surfaces: The touching process and effects of macro- and microsurface structure. Perception \& Psychophysics, 16(2), 385-395.

Lederman, S. J., Klatzky, R. L., Hamilton, C. L., \& Ramsay, G. I. (1999). Perceiving roughness via a rigid probe: Psychophysical effects of exploration speed and mode of touch. Haptics-e: The Electronic Journal for Haptics Research, 1(1), http://www.haptics-e.org.

Lederman, S. J., \& Taylor, M. M. (1972). Fingertip force, surface geometry, and the perception of roughness by active touch. Perception \& Psychophysics, 12(5), $401-408$.

Lederman, S. J., Thorne, G., \& Jones, B. (1986). Perception of texture by vision and touch: Multidimensionality and intersensory integration. Journal of Experimental Psychology: Human Perception and Performance, 12(2), 169-180. 
Macmillan, N. A., \& Creelman, C. D. (2004). Detection Theory: A User's Guide (2nd ed.). New York: Lawrence Erlbaum Associates.

Massie, T. H. (1996). Initial haptic explorations with the PHANToM: Virtual touch through point interaction. Unpublished Master's Thesis, Massachusetts Institute of Technology.

Nefs, H. T., Kappers, A. M. L., \& Koenderink, J. J. (2001). Amplitude and spatial-period discrimination in sinusoidal gratings by dynamic touch. Perception, 30, 1263-1274.

Nefs, H. T., Kappers, A. M. L., \& Koenderink, J. J. (2003). Detection of amplitude modulation and frequency modulation in tactual gratings: A critical bandwidth for active touch. Perception, 32(10), 1259-1271.

O'Malley, M. K., \& Goldfarb, M. (2005). On the ability of humans to haptically identify and discriminate real and simulated objects. PRESENCE: Teleoperators and Virtual Environments, 14(3), 366376.

Pang, X. D., Tan, H. Z., \& Durlach, N. I. (1991). Manual discrimination of force using active finger motion. Perception \& Psychophysics, 49(6), 531-540.

Poling, G. L., \& Weisenberger, J. M. (2004). Multisensory roughness perception of virtual surfaces: effects of correlated cues. Proceedings of the 12th International Symposium on Haptic Interfaces for Virtual Environment and Teleoperator Systems (HAPTICS '04), 161- 168.

Rosenberg, L. B., \& Adelstein, B. D. (1993). Perceptual Decomposition of Virtual Haptic Surfaces, Proceedings of the IEEE Symposium on Research Frontiers in Virtual Reality (pp. 46-53). San Jose, CA.

Steger, R., Lin, K., Adelstein, B. D., \& Kazerooni, H. (2004). Design of a passively balanced spatial linkage haptic interface. ASME J. Mech. Des., 126, 984-991.

Stevens, S. S., \& Harris, J. R. (1962). The scaling of subjective roughness and smoothness. Journal of Experimental Psychology, 64(5), 489-494.

Traylor, R., Wilhelm, D., Adelstein, B. D., \& Tan, H. Z. (2005). Design considerations for stand-alone haptic interfaces communicating via UDP protocol, Proceedings of the 2005 World Haptics Conference
(WHC05): The First Joint EuroHaptics Conference and the Symposium on Haptic Interfaces for Virtual Environment and Teleoperator Systems (pp. 563-564). Pisa, Italy, Mar. 18-20.

Unger, B. J., Nicolaidis, A., Berkelman, P. J., Thompson, A., Lederman, S., Klatzky, R. L., \& Hollis, R. L. (2002). Virtual peg-in-hole performance using a 6-DOF magnetic levitation haptic device: Comparison with real forces and with visual guidance alone. Proceedings of the 10th International Symposium on Haptic Interfaces for Virtual Environment and Teleoperator Systems, 263270.

Weisenberger, J. M., \& Krier, M. J. (1997). Haptic perception of simulated surface textures via vibratory and force feedback displays. Proceedings of the ASME Dynamic Systems and Control Division, 61, 55-60.

Weisenberger, J. M., Krier, M. J., \& Rinker, M. A. (1998). Resolution of virtual grating orientation with 2-DOF and 3-DOF force feedback systems. In R. J. Furness (Ed.), Proceedings of the ASME Dynamic Systems and Control Division (Vol. 64, pp. 295-301). New York: ASME.

Weisenberger, J. M., Krier, M. J., \& Rinker, M. A. (2000). Judging the orientation of sinusoidal and square-wave virtual gratings presented via 2-DOF and 3-DOF haptic interfaces. Haptics-e, 1(4), http://www.haptics-e.org.

Weisenberger, J. M., Krier, M. J., Rinker, M. A., \& Kreidler, S. M. (1999). The role of the end-effector in the perception of virtual surfaces presented via force-feedback haptic interfaces. In N. Olgac (Ed.), Proceedings of the ASME Dynamic Systems and Control Division (Vol. 67, pp. 35-41): ASME.

West, A. M., \& Cutkosky, M. R. (1997). Detection of real and virtual fine surface features with a haptic interface and stylus. In G. Rizzoni (Ed.), Proceedings of the ASME Dynamic Systems and Control Division (Vol. 61, pp. 159-166). New York: ASME. 